\title{
An Evolutionary SPDE Breeding-Based Hybrid Particle Swarm Optimizer: Application in Coordination of Robot Ants for Camera Coverage Area Optimization
}

\author{
Debraj De ${ }^{1}$, Sonai Ray ${ }^{1}$, Amit Konar $^{1}$, and Amita Chatterjee ${ }^{2}$ \\ ${ }^{1}$ Department of Electronics and Telecommunication Engineering, Jadavpur University, \\ Kolkata- 700032, India \\ debraj00@rediffmail.com, ssonai2003@yahoo.com, \\ amit_konar@vsnl.net \\ ${ }^{2}$ Centre of Cognitive Science, Jadavpur University, \\ Kolkata- 700032, India \\ amita_juayahoo.com
}

\begin{abstract}
In this paper we propose a new Hybrid Particle Swarm Optimizer model based on particle swarm, with breeding concepts from novel evolutionary algorithms. The hybrid PSO combines traditional velocity and position update rules of RANDIW-PSO and ideas from Self Adaptive Pareto Differential Evolution Algorithm (SPDE). The hybrid model is tested and compared with some high quality PSO models like the RANDIW-PSO and TVIW-PSO. The results indicate two good prospects of our proposed hybrid PSO model: potential to achieve faster convergence as well as potential to find a better solution. The hybrid PSO model, with the abovementioned features, is then efficiently utilized to coordinate robot ants in order to help them to probe as much camera coverage area of some planetary surface or working field as possible with minimum common area coverage.
\end{abstract}

\section{Introduction}

Particle Swarm Optimization (PSO) is a population based self-adaptive search optimization technique, first introduced by Kennedy and Eberhart [1] in 1995. One of the latest promising quality models in PSO technique is the Particle Swarm Optimizer with Random Inertia Weight (RANDIW-PSO model). Evolutionary algorithms [5] are a kind of global optimization techniques that use selection and recombination as their primary operators to tackle optimization problems. Differential evolution (DE) is a branch of evolutionary algorithms developed by Rainer Storn and Kenneth Price for optimization problems over continuous domains. Of the latest Pareto Differential Evolution (PDE) algorithms, the Self-Adaptive Pareto Differential Evolution Algorithm (SPDE) [6] has the important property to self adapt the crossover and mutation rates.

The proposed hybrid PSO model incorporates crossover which is motivated by SPDE evolution. Here for some randomly selected dimensions particles are reselected and refined from SPDE crossover pool and goes under position updating. The paper is organized as follows: section 2 presents proposed hybrid PSO model, results and comparisons with some other PSO models are exhibited in section 3 and finally the 
hybrid PSO model is applied to solve coordination problem of robot ants which are engaged in covering some planetary surface by their camera, such that the common area coverage is minimum.

\section{New Hybrid PSO Model}

Here we propose a Hybrid Random Inertia Weight (RANDIW)-PSO model, in which breeding is performed by the Self Adaptive Pareto Differential Evolution (SPDE). The total algorithm is illustrated as follows:
A. Randomly generate the initial population.
B. Repeat
until number of generation reaches its maximum limit:
1. Randomly generate $m$ number of dimensions that will be bred.
2. For $i=1$ to $m$, do

a. Construct SPDE breeding pools, each of which contain position values of $i$ th dimension $\left(x_{i}\right)$ of three particles, chosen randomly.

b. In each of the breeding pools, do

i. Mark the minimum of three $x_{i}$ values as the main parent $\alpha_{1}$. Other two are supporting parent $\alpha_{2}$ and $\alpha_{3}$.

ii. The crossover rate of each individual is chosen as $\left(1-x_{i j}\right)$, where $x_{i j}^{\prime}$ is the normalized position value of $i$-th dimension of $j$-th particle.

iii. Calculate Crossover rate $\left(x_{c}\right)$ of child:

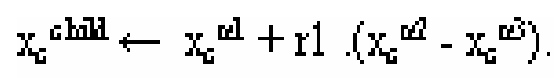

Where $\mathrm{r} 1$ is a random number $[0,1]$.

iv. Crossover: Select a random variable $\mathrm{j}[1,3]$

For each variable $k$ (value 1 or 2 or 3 )

With some random probability $[0,1]>x_{c}^{\text {child }}$ or if $k=j$,

do

Crossover between that $\alpha_{k}$ and the main parent $\alpha_{1}$.

Crossover is performed by arithmetic crossover on the position of the parents as follows:

$$
\begin{aligned}
& \operatorname{child}_{1}\left(\mathrm{x}_{\mathrm{i}}\right)=\mathrm{p}_{\mathrm{i}} \cdot \operatorname{parent}_{1}\left(\mathrm{x}_{\mathrm{i}}\right)+\left(1.0-\mathrm{p}_{\mathrm{i}}\right) \cdot \operatorname{parent}_{2}\left(\mathrm{x}_{\mathrm{i}}\right) . \\
& \operatorname{child}_{2}\left(\mathrm{x}_{\mathrm{i}}\right)=\mathrm{p}_{\mathrm{i}} \cdot \operatorname{parent}_{2}\left(\mathrm{x}_{\mathrm{i}}\right)+\left(1.0-\mathrm{p}_{\mathrm{i}}\right) \cdot \operatorname{parent}_{1}\left(\mathrm{x}_{\mathrm{i}}\right) .
\end{aligned}
$$

Where $\mathrm{p}_{\mathrm{i}}$ is uniformly distributed random value between 0 and 1 . The velocity of the offspring is calculated as the sum of velocity vectors of the parent normalized to the original length of each parent velocity vector.

$$
\begin{gathered}
\operatorname{child}_{1}(\mathrm{v})=\left[\left\{\operatorname{parent}_{1}(\mathrm{v})+\underset{\left.\left.\operatorname{parent}_{2}(\mathrm{v})\right\} /\left|\operatorname{parent}_{1}(\mathrm{v})+\operatorname{parent}_{2}(\mathrm{v})\right|\right] . \mid}{\operatorname{parent}_{1}(\mathrm{v}) \mid}\right.\right. \\
\operatorname{child}_{2}(\mathrm{v})=\left[\left\{\operatorname{parent}_{1}(\mathrm{v})+\underset{\left.\left.\operatorname{parent}_{2}(\mathrm{v})\right\} /\left|\operatorname{parent}_{1}(\mathrm{v})+\operatorname{parent}_{2}(\mathrm{v})\right|\right] . \mid}{\operatorname{parent}_{2}(\mathrm{v}) \mid .}\right.\right.
\end{gathered}
$$


The arithmetic crossover of positions and velocity vectors used were empirically tested to be the most promising. The arithmetic crossover of positions in the search space is one of the most commonly used crossover methods with standard real valued GAs, placing the offspring within the hypercube spanned by the parent particles.

iv. After the crossover, from the three parent and children, three minimum individuals are retained.

3. Apply PSO equation: The PSO equation follows the TVIW-PSO model:

$$
\begin{gathered}
\mathrm{v}_{\mathrm{i}}=\mathrm{w} \cdot \mathrm{v}_{\mathrm{i}}+\varphi_{1 \mathrm{i}} \cdot\left(\mathrm{p}_{\mathrm{i}}-\mathrm{x}_{\mathrm{i}}\right)+\varphi_{2 \mathrm{i}} \cdot\left(\mathrm{p}_{\mathrm{g}}-\mathrm{x}_{\mathrm{i}}\right) . \\
\mathrm{x}_{\mathrm{i}}=\mathrm{x}_{\mathrm{i}}+\mathrm{v}_{\mathrm{i}} .
\end{gathered}
$$

Where the inertia weight $w$ is set to change randomly according to the following equation:

$$
\mathrm{W}=0.5+\{\operatorname{rand}(.) / 2\} .
$$

The term rand(.) is a uniformly distributed random number within the range $[0,1]$, thus with mean value $0.75 . \varphi_{1 \mathrm{i}}$ and $\varphi_{2 \mathrm{i}}$ are random values different for each particle and for each dimension. If the velocity is higher than a certain limit, called $v_{\max }$, this limit will be used as the new velocity for this particle in this dimension, thus keeping the particles within search space.

\section{Experimental Results}

Our proposed SPDE breeding based hybrid RANDIW-PSO was compared with basic RANDIW-PSO and TVIW-PSO models on five standard test functions and it exhibited two important striking features of proposed hybrid PSO: faster convergence and resulting better solution, as illustrated clearly by the following tables and graphs.

Table 1. Initialization range, dynamic range and maximum for benchmarks

\begin{tabular}{|c|c|c|c|}
\hline Test function & Range of search & $\begin{array}{c}\text { Range of } \\
\text { initialization }\end{array}$ & $\mathrm{v}_{\max }$ \\
\hline Sphere (f1) & $(-100,100)^{\mathrm{n}}$ & $(50,100)^{\mathrm{n}}$ & 100 \\
\hline Rosenbrock (f2) & $(-100,100)^{\mathrm{n}}$ & $(15,30)^{\mathrm{n}}$ & 100 \\
\hline Rastrigrin (f3) & $(-10,10)^{\mathrm{n}}$ & $(2.56,5.12)^{\mathrm{n}}$ & 10 \\
\hline Griewank (f4) & $(-600,600)^{\mathrm{n}}$ & $(300,600)^{\mathrm{n}}$ & 600 \\
\hline Schaffer (f6) & $(-100,100)^{2}$ & $(15,30)^{2}$ & 100 \\
\hline
\end{tabular}


Table 2. Average value of the benchmarks for 50 trials

\begin{tabular}{|c|c|c|c|c|c|}
\hline Function & Dimen & Generation & \multicolumn{3}{|c|}{ Average } \\
\cline { 4 - 6 } & sion & & $\begin{array}{c}\text { PSO- } \\
\text { RANDIW }\end{array}$ & PSO-TVIW & $\begin{array}{c}\text { Proposed SPDE } \\
\text { based hybrid } \\
\text { PSO }\end{array}$ \\
\hline \multirow{2}{*}{ f1 } & 10 & 1000 & 0.01 & 0.01 & 0.01 \\
\cline { 2 - 6 } & 20 & 2000 & 0.01 & 0.01 & 0.01 \\
\cline { 2 - 6 } & 30 & 3000 & 0.01 & 0.01 & 0.01 \\
\hline \multirow{2}{*}{ f2 } & 10 & 3000 & 2.212 & 26.840 & 2.002 \\
\cline { 2 - 6 } & 20 & 4000 & 28.332 & 53.653 & 26.708 \\
\cline { 2 - 6 } & 30 & 5000 & 38.227 & 63.352 & 36.586 \\
\hline \multirow{3}{*}{ f3 } & 10 & 3000 & 1.001 & 1.989 & 1.989 \\
\cline { 2 - 6 } & 20 & 4000 & 19.984 & 3.979 & 8.944 \\
\cline { 2 - 6 } & 30 & 5000 & 44.772 & 15.919 & 10.823 \\
\hline f4 & 10 & 3000 & 0.0372 & 0.0765 & 0.0276 \\
\cline { 2 - 6 } & 20 & 4000 & 0.0074 & 0.0812 & 0.0070 \\
\cline { 2 - 6 } & 30 & 5000 & 0.0932 & 0.1048 & 0.01478 \\
\hline f6 & 2 & 100 & 0.00247 & 0.00246 & 0.00240 \\
\hline
\end{tabular}

\section{References}

1. Kennedy, J., Eberhart, R.: Particle Swarm Optimization. Proc. IEEE Int. Conf. Neural Networks (1995) 1942-1948

2. Ratnaweera, A., Halgamuge, S.K., Watson, H.C.: Self-Organizing Hierarchical Particle Swarm Optimizer with Time-Varying Acceleration Coefficients. IEEE Transactions On Evolutionary Computation, Vol.8. (2004)

3. Kennedy, J.: The Particle Swarm: Social Adaptation of Knowledge. Proc. IEEE Int. Conf. Evolutionary Computation (1997) 303-308

4. Lovbjerg, M., Rasmussen, T.K., Krink, T.: Hybrid Particle Swarm Optimizer with Breeding and Subpopulation. Proc. 3rd Genetic Evolutionary Computation Conf. (GECCO-2001), San Fransisco, CA (2001) 469-476

5. Coello, C.A.: A Comprehensive Survey of Evolutionary-based Multiobjective Optimisation Techniques. Knowledge and Information Systems (1999) 269 - 308 